\title{
Analysis of multiwalled carbon nanotubes as waveguides and antennas in the infrared and the visible regimes
}

\author{
M. V. Shuba, G. Ya. Slepyan, S. A. Maksimenko \\ Institute for Nuclear Problems, Belarus State University, Bobruiskaya 11, 220050 Minsk, Belarus \\ C. Thomsen \\ Institut für Festkörperphusik, Technische Universität Berlin, Hardenbergstr. 36, D-10623 Berlin, Germany \\ A. Lakhtakia \\ Nanoengineered Metamaterials Group, Department of Engineering Science and Mechanics, \\ Pennsylvania State University, University Park, PA 16802-6812, USA
}

\begin{abstract}
The propagation of azimuthally symmetric guided waves in multiwalled carbon nanotubes (MWCNTs) was analyzed theoretically in the mid-infrared and the visible regimes. The MWCNTs were modeled as ensembles of concentric, cylindrical, conducting shells. Slightly attenuated guided waves and antenna resonances due to the edge effect exist for not-too-thick MWCNTs in the far- and mid-infrared regimes. Interband transitions hinder the propagation of guided waves and have a deleterious effect on the performance of a finite-length MWCNT as an antenna. Propagation of surface-plasmon waves along an MWCNT with a gold core was also analyzed. In the near-infrared and the visible regimes, the shells behave effectively as lossy dielectrics suppressing surface-plasmonwave propagation along the gold core.
\end{abstract}

PACS numbers: 42.70.-a, 73.25.+i, 77.84.Lf, 78.67.Ch

\section{INTRODUCTION}

Their unusual physical and chemical properties, and their potential applications in a variety of nanotechnologies make carbon nanotubes (CNTs) very in-

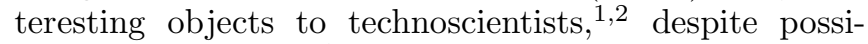
ble health hazards $\stackrel{3}{3}$ In particular, CNTs have been proposed to fabricate several different integratedcircuits elements and electromagnetic devices, such as transmission lines, $, 4,5,6,7,8$ interconnects, $, 9,10,11,12$ and nanoantennas. $13,14,15,16,17,18,19,20$ The fabrications of a CNT-based amplitude modulator/demodulator ${ }^{21}$ and a fully integrated radio receiver ${ }^{22}$ have been reported. References 23,24,25,26,27,28,29 demonstrate the potential of CNTs as emitters of terahertz and infrared radiation.

Not surprisingly, the electromagnetic characteristics of CNT-based antennas have been examined in different frequency regimes ranging from the microwave to the visible. CNT morphology has been demonstrated to play a crucial role, as evinced by reported research on single-wall CNTs (SWCNTs) $, 14,15,16,30 \sim 1-$ cm-long one-dimensional chains of electrically connected

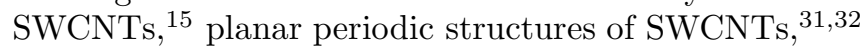
CNT bundles ${ }^{33,34}$, and CNT arrays ${ }^{35}$ Continuing in that vein, here we report our work on the performance of multiwalled CNTs (MWCNTs) as antennas.

A multiwalled CNT comprises $N$ concentric shells (or tubes), each obtained by rolling a graphene sheet into a cylinder. The number of shells in a MWCNT can range from 2 to 200, and the distance between consecutive shells from 3.4 to $3.6 \AA, 36$ which is close to the interlayer distance in graphite $(3.35 \AA)$. The lattice structures of consecutive shells are generally uncorrelated with each other, and can even have different chiralities. In fact, several experiments on MWCNTs have indicated that often the different shells have different periodicities $\underline{37,38}$ Two consecutive shells of an MWCNT are called commensurate (incommensurate) if the ratio of their unitcell lengths along the CNT axis is rational (irrational), indicating the presence (absence) of translational symmetry. Incommensurability affects the transport and optical

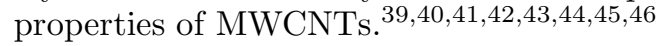

The critical issue when modeling the electromagnetic properties of an MWCNT is the intershell interaction leading to intershell electron tunneling or hopping. Published data, although very variable, shows a strong dependence on the intrinsic symmetries of the shells, which dictates selection rules for the elements of the tunneling matrix, as determined by the conservation laws for energy and momentum. As may be expected, two incommensurate shells interact differently than two commensurate

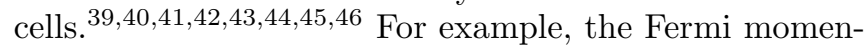
tums of two incommensurate shells do not coincide within the first Brillouin zone and therefore the intershell tunneling vanishes $\stackrel{43}{ }$

An isolated MWCNT can be modeled in several different ways. Abrikosov et al $\underline{\underline{47}}$ considered an MWCNT as a set of coaxial, continuous, conducting cylinders accompanied by an appropriate Kronig-Penney-type potential in the radial direction. Dyachkov and Makaev $\underline{48}$ as well as Tunney and Cooper $\stackrel{49}{ }$ assumed the intershell interaction to be so small that each shell could be considered to be in a perturbed eigenstate of an SWCNT. A computer simulation with some input parameters extracted from experiment has also been reported $\underline{\underline{50}}$

Following Refs. 48 and 49, we decided to neglect the contribution of the intershell tunneling to the radiation 
characteristics of an MWCNT. Indeed, though intershell interaction in defectless finite-length double-wall CNTs shifts optical band gaps and distorts the density of electronic states, it does not subvert the intrinsic type of conductivity (either metallic or semiconductor) $; 47,48,49,50$ therefore, it would influence the radiation characteristics only slightly. As the presence of localized defects can dramatically increase intershell conduction, $\stackrel{49}{\underline{4}}$ we restrict ourselves here to MWCNTs with defect-free shells by neglecting intershell conduction.

Analysis of the physical characteristics of CNT-based integrated circuits elements, such as antennas, must follow the general principles of electrodynamics and must account for the peculiar dispersion properties of electrons in CNTs. A key element of the analysis is the formulation of the effective boundary conditions for the electro-

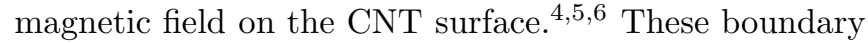
conditions are dictated by the microscopic model of CNT used. Hence, any attempt to describe an MWCNT with cross-sectional radius between 25 and $150 \mathrm{~nm}$ as a perfectly conducting rod 13,35 can provide only rough estimates, and is inapplicable to MWCNTs of small radius.

We adopt a proper microscopic model for MWCNTs here. A relevant boundary-value problem for an MWCNT of finite length is formulated in Sec. III. Since the radiation characteristics of an MWCNT are determined by its waveguiding properties, the dispersion equation for guided-wave propagation on an infinitely long MWCNT is derived in Sec. III. The boundary-value problem of Sec. II is solved numerically by an integralequation technique in Sec. IV] This approach is well established in antenna theory ${ }^{51}$ and has been successfully applied to SWCNT antennas $\frac{14}{4}$ and almost circu-

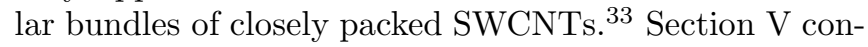
tains numerical results for guided-wave parameters (slowwave and attenuation) and the scattering properties of MWCNT in a wide frequency range from the terahertz to the visible regimes. Sections VI presents an assessment of the potential of an isolated MWCNT function as an optical nanoantenna. Concluding remarks are provided in Sec. VII] An $\exp (-i \omega t)$ time-dependence is implicit, $t$ denotes the time, and $\omega$ is the angular frequency.

\section{MODEL}

Let us model an MWCNT as a multishell structure comprising $N$ coaxial, infinitesimally thin cylinders in free space. Each shell is achiral, i.e., it has either a zigzag $(m, 0)$ or an armchair $(m, m)$ configuration, with $m$ as an integer. Therefore, our model is applicable to both semiconducting and metallic shells. The sequence of armchair and zigzag shells in the MWCNT is arbitrary.

Let the cylindrical axis of the chosen MWCNT be par- allel to the $z$ axis of the cylindrical coordinate system $(\rho, \phi, z)$. The centroid of the MWCNT is assumed to coincide with the origin of the coordinate system.

Let us enumerate shells in the MWCNT consecutively from 1 to $N$ beginning from the innermost shell, so that their cross-sectional radii comply with the condition $R_{N}>R_{N-1}>\ldots>R_{1}$. The cross-sectional radius $R_{N}$ of the outermost shell is assumed to be much smaller than the free-space wavelength. The $p$-th shell, $p \in[1, N]$, possesses an axial conductivity per unit area denoted by $\sigma_{p}$. The transverse current density in every shell is neglected. Intershell tunneling is also neglected, as mentioned in the previous section.

As electromagnetic fields with azimuthal symmetry are easily excited in an MWCNT by a uniform external field, we decided to restrict ourselves to azimuthally symmetric fields. This restriction also holds for finite-length MWCNTs in the long-wavelength regime (Sec. $\mathrm{VB}$ ). The electric Hertz vector $\Pi \equiv \Pi(\rho, z) \mathbf{e}_{z}$ is then governed by the Helmholtz equation

$$
\frac{1}{\rho} \frac{\partial}{\partial \rho}\left(\rho \frac{\partial \Pi}{\partial \rho}\right)+\frac{\partial^{2} \Pi}{\partial z^{2}}+k^{2} \Pi=0
$$

where $\mathbf{e}_{z}$ is the unit vector along the $z$ axis, $k=\omega / c$ the free-space wavenumber, and $c$ is speed of light in free space (i.e., vacuum). Since $\Pi$ depends only on $\rho$ and $z$, the components of the electric and magnetic fields are as follows:

$$
\begin{gathered}
E_{\rho}=\frac{\partial^{2} \Pi}{\partial \rho \partial z}, E_{\phi}=0, E_{z}=\left(\frac{\partial^{2}}{\partial z^{2}}+k^{2}\right) \Pi, \\
H_{\rho}=0, H_{\phi}=i k \frac{\partial \Pi}{\partial \rho}, H_{z}=0 .
\end{gathered}
$$

On neglecting intershell tunneling, the two boundary conditions across the $p$-th shell are given by ${ }^{\underline{4}}$

$$
\begin{gathered}
\left.\frac{\partial \Pi}{\partial \rho}\right|_{\rho=R_{p}+0}-\left.\frac{\partial \Pi}{\partial \rho}\right|_{\rho=R_{p}-0}=\frac{4 \pi}{i k c} J_{p}, \\
\left.\Pi\right|_{\rho=R_{p}+0}=\left.\Pi\right|_{\rho=R_{p}-0} .
\end{gathered}
$$

Here $\mathbf{J}_{p}(z)=J_{p}(z) \mathbf{e}_{z}$ is the axial current density on the surface $\rho=R_{p}$, with

$$
J_{p}(z)=\sigma_{p}\left(\frac{d^{2}}{d z^{2}}+k^{2}\right) \Pi\left(R_{p}, z\right) .
$$

The surface conductivity $\sigma_{p}$ of the $p$-th isolated shell is available via quantum-transport calculations carried out in the tight-binding approximation as $\$ .52$ 


$$
\sigma_{p}(\omega)=-\frac{i e^{2} \omega}{\pi^{2} \hbar R_{p}}\left\{\frac{1}{\omega(\omega+i / \tau)} \sum_{s=1}^{m} \int_{1 s t B Z} \frac{\partial F_{c}}{\partial p_{z}} \frac{\partial \mathcal{E}_{c}}{\partial p_{z}} d p_{z}-2 \sum_{s=1}^{m} \int_{1 s t B Z} \mathcal{E}_{c}\left|R_{v c}\right|^{2} \frac{F_{c}-F_{v}}{\hbar^{2} \omega(\omega+i / \tau)-4 \mathcal{E}_{c}^{2}} d p_{z}\right\}
$$

where $e$ is the electron charge, $\hbar$ is the normalized Planck constant, and $p_{z}$ is the axial projection of quasimomentum of an electron. The integer $s \in[1, m]$ labels the $\pi$-electron energy bands and the abbreviation 1 st $B Z$ restricts the variable $p_{z}$ to the first Brillouin zone. The time constant $\tau$ (being the relaxation time) is assumed to be equal to the inverse of the relaxation frequency $\nu$. The normalized matrix elements of the dipole transition between the conduction and valence bands, denoted by $R_{v c}$, are evaluated in the tight-binding approximation, after taking into account the transverse quantization of the charge carriers' motion and the hexagonal structure of the graphene lattice. $\underline{6}$

The first term on the right side of (7) is the Drude term corresponding to the intraband conductivity, while the second term describes the contribution of interband transitions between the valence and the conduction bands. The indexes $c$ and $v$ refer to the conduction and valence bands, respectively. The equilibrium Fermi distribution functions

$$
F_{c}\left(p_{z}, s\right)=\left\{1+\exp \left[\frac{\mathcal{E}_{c}\left(p_{z}, s\right)}{k_{B} T}\right]\right\}^{-1}
$$

and

$$
F_{v}\left(p_{z}, s\right)=\left\{1+\exp \left[\frac{\mathcal{E}_{v}\left(p_{z}, s\right)}{k_{B} T}\right]\right\}^{-1}
$$

involve the temperature $T$ and the Boltzmann constant $k_{B}$. The electron energies $\mathcal{E}_{c, v}\left(p_{z}, s\right)$ and normalized matrix elements of the dipole transition $R_{v c}$ for zigzag $(m, 0)$ CNT are given by 1

$$
\begin{aligned}
\mathcal{E}_{c}\left(p_{z}, s\right) & =-\mathcal{E}_{v}\left(p_{z}, s\right) \\
& =\gamma_{0} \sqrt{1+4 \cos \left(a p_{z}\right) \cos \left(\frac{\pi s}{m}\right)+4 \cos ^{2}\left(\frac{\pi s}{m}\right)}
\end{aligned}
$$

and

$$
\begin{aligned}
& R_{v c}\left(p_{z}, s\right)=-\frac{b \gamma_{0}^{2}}{2 \mathcal{E}_{c}^{2}} \\
& \quad \times\left[1+\cos \left(a p_{z}\right) \cos \left(\frac{\pi s}{m}\right)-2 \cos ^{2}\left(\frac{\pi s}{m}\right)\right],
\end{aligned}
$$

respectively, where $\gamma_{0} \approx 2.7 \mathrm{eV}$ is the overlap integral, 1 $a=3 b / 2 \hbar$, and $b=0.142 \mathrm{~nm}$ is the interatomic distance in graphene. Expressions for $\mathcal{E}_{c}\left(p_{z}, s\right)$ and $R_{v c}\left(p_{z}, s\right)$ for armchair $(m, m)$ CNTs are available elsewhere $\underline{\underline{1}}$

\section{GUIDED-WAVE PROPAGATION IN AN INFINITELY LONG MWCNT}

Let us recall that intershell tunneling is negligibly small so that $\Pi$ can be represented as a superposition of $N$ independent functions; furthermore, we seek a solution of (11) in the form of a guided wave. Accordingly,

$$
\Pi(\rho, z)=e^{i h z} \sum_{p=1}^{N} A_{p} \Phi_{p}(\rho)
$$

where $\left\{A_{p}\right\}$ is the set of unknown coefficients and $h$ is the unknown guide wavenumber. With $\kappa=\sqrt{h^{2}-k^{2}}$, the basis function $\Phi_{p}(\rho)$ is taken to satisfy the differential equation

$$
\frac{1}{\rho} \frac{d}{d \rho}\left[\rho \frac{d}{d \rho} \Phi_{p}(\rho)\right]+\kappa^{2} \Phi_{p}(\rho)=0, \quad p \in[1, N],
$$

subject to the following boundary conditions at the surface $\rho=R_{p}$ :

$$
\begin{gathered}
\left.\frac{\partial}{\partial \rho} \Phi_{p}(\rho)\right|_{\rho=R_{p}+0}-\left.\frac{\partial}{\partial \rho} \Phi_{p}(\rho)\right|_{\rho=R_{p}-0}=\frac{4 \pi}{i k c} \\
\left.\Phi_{p}(\rho)\right|_{\rho=R_{p}+0}=\left.\Phi_{p}(\rho)\right|_{\rho=R_{p}-0}
\end{gathered}
$$

The appropriate solution of (13) is

$$
\Phi_{p}(\rho)=\frac{4 i \pi R_{p}}{k c} \begin{cases}K_{0}\left(\kappa R_{p}\right) I_{0}(\kappa \rho), & \rho<R_{p}, \\ I_{0}\left(\kappa R_{p}\right) K_{0}(\kappa \rho), & \rho>R_{p}\end{cases}
$$

where $I_{0}(\cdot)$ and $K_{0}(\cdot)$ are modified Bessel functions of the zeroth order.

Whereas the boundary condition (5) is automatically satisfied by (12) and (16), (4) remains to be satisfied. Substitution of (12) into (4) leads to a system of $N$ linear homogeneous equations with respect to the coefficients $A_{p}$. A nontrivial solution of the system is provided by the dispersion equation

$$
\operatorname{det} \mathrm{M}=0 \text {. }
$$

The elements $M_{q p}$ of the $N \times N$ matrix $\mathrm{M}$ are given by

$$
M_{q p}=\left\{\begin{array}{l}
K_{0}\left(\kappa R_{q}\right) I_{0}\left(\kappa R_{p}\right), \quad q>p \\
K_{0}\left(\kappa R_{p}\right) I_{0}\left(\kappa R_{q}\right)-\frac{i \omega \delta_{q p}}{4 \pi R_{q} \sigma_{q} \kappa^{2}}, \quad q \leq p
\end{array}\right.
$$

where $\delta_{q p}$ is the Kronecker delta. The dispersion equation (17) has $N \kappa$-roots, each corresponding to a guided wave in the MWCNT. From each $\kappa$-root, we can determine $h$ and the slow-wave coefficient $\beta=k / h$. 


\section{LIGHT SCATTERING BY A FINITE-LENGTH MWCNT}

\section{A. Integral-equation technique}

Let us now consider the scattering of an electromagnetic wave incident on a MWCNT of finite length $L$. The scattered field can be described by (2) and (3) with the electric Hertz vector satisfying the Helmholz equation (1), the usual radiation condition, 51 and the boundary conditions (4) and (5). The surface current density $J_{p}(z)$ at $\rho=R_{p}$ is given by

$$
J_{p}(z)=\sigma_{p}\left(\frac{d^{2}}{d z^{2}}+k^{2}\right) \Pi\left(R_{p}, z\right)+\sigma_{p} E_{z}^{(0)}\left(R_{p}, z\right),
$$

where $E_{z}^{(0)}(\rho, z)$ is the $z$-component of the incident electric field. As the intershell tunneling through the two ends of the MWCNT is negligible, the current density $J_{p}(z)$ satisfies the edge conditions

$$
J_{p}( \pm L / 2)=0
$$

thereby expressing the absence of concentrated charges on the ends. The boundary-value problem can effectively be solved by the integral-equation technique for the surface current density as follows. ${ }^{53,54}$

The potential $\Pi(\rho, z)$ must be linearly related to $J_{p}(z)$, $p \in[1, N]$, as

$$
\Pi(\rho, z)=\frac{i}{\omega} \sum_{p=1}^{N} R_{p} \int_{-L / 2}^{L / 2} J_{p}\left(z^{\prime}\right) G\left(z-z^{\prime}, \rho, R_{p}\right) d z^{\prime},
$$

where

$$
G(z, \rho, R)=\int_{0}^{2 \pi} \frac{\exp \left(i k \sqrt{\rho^{2}+R^{2}-2 R \rho \cos \varphi+z^{2}}\right)}{\sqrt{\rho^{2}+R^{2}-2 R \rho \cos \varphi+z^{2}}} d \varphi
$$

is the free-space Green function for (1). Setting $\rho=R_{q}$ in (21) and making use of (19), we arrive at the following system of $N$ integral equations with respect to unknown current densities:

$$
\begin{aligned}
& -\frac{1}{2 i k} \int_{-L / 2}^{L / 2} E_{z}^{(0)}\left(R_{q}, z^{\prime}\right) \exp \left(i k\left|z-z^{\prime}\right|\right) d z^{\prime} \\
& \quad+C_{q} \exp (i k z)+D_{q} \exp (-i k z) \\
& =\sum_{p=1}^{N} \int_{-L / 2}^{L / 2}\left[\frac{2 \pi i R_{p}}{\omega} G\left(z-z^{\prime}, R_{p}, R_{q}\right)\right. \\
& \left.\quad+\frac{i \delta_{q p}}{2 k \sigma_{p}} \exp \left(i k\left|z-z^{\prime}\right|\right)\right] J_{p}\left(z^{\prime}\right) d z^{\prime}, \quad q \in[1, N] .
\end{aligned}
$$

Here, $C_{q}$ and $D_{q}$ are unknown constants to be determined from the edge conditions (20).
In the long-wavelength regime $(\lambda \gg L)$, the electromagnetic scattering properties of the MWCNT can be encapsulated in a polarizability tensor with only one nonzero component

$$
\alpha_{z z}=\frac{2 \pi i}{\omega E_{z}^{(0)}(0,0)} \sum_{p=1}^{N} R_{p} \int_{-L / 2}^{L / 2} J_{p}(z) d z .
$$

The integral on the right side of (23) can be numerically handled by a quadrature formula. ${ }^{55}$ Parenthetically, the foregoing formalism was recently applied to almost circular, closely packed bundles of finite-length parallel, identical, metallic SWCNTs 33

A finite-length MWCNT functioning as a receiving antenna can be characterized by the antenna efficiency ${ }^{33}$

$$
\eta=\frac{P_{r}}{P_{t}+P_{r}}
$$

where

$$
P_{r}=\frac{\pi^{2} k^{2}}{c} \int_{0}^{\pi} \sin ^{3} \theta\left|\int_{-L / 2}^{L / 2} e^{i k z \cos \theta} \sum_{p=1}^{N} R_{p} J_{p}(z) d z\right|^{2} d \theta
$$

is the scattered power and

$$
P_{t}=\pi \sum_{p=1}^{N} R_{p} \operatorname{Re}\left(\frac{1}{\sigma_{p}}\right) \int_{-L / 2}^{L / 2}\left|J_{p}(z)\right|^{2} d z
$$

is the power lost to ohmic dissipation.

\section{B. Interband-transitions regime: first Born approximation}

According to Refs. 16 and 32 , surface waves in a CNT are strongly attenuated in the frequency regime of interband transitions. Therefore, the surface current density $J(z)$ in an SWCNT of radius $R$ exposed to an incident electric field $\mathbf{E}^{(0)}$ obeys Ohm's law

$$
J(z)=\sigma E_{z}^{(0)}(R, z)
$$

very well, $\sigma$ being the surface conductivity. Equation (28) can be considered as the first Born approximation. 56

Application of the first Born approximation (28) to an MWCNT allows us to express the axial surface current density $J_{p}(z)$ on the surface of the $p$-th shell as

$$
J_{p}(z) \approx \sigma_{p} E_{z}^{(0)}\left(R_{p}, z\right), \quad p \in[1, N] .
$$

This expression can be justified as follows. The electric field exciting the $p$-th shell is made of two components: (i) the electric field $\mathbf{E}^{(0)}$ incident on the entire MWCNT and (ii) the sum of the electric fields radiated to the surface current densities $J_{q}, q \in[1, N]$ but $q \neq p$. Equation (29) is justified if the first component is much larger 
than the second component at $\rho=R_{p}$, i.e., the condition

$$
\begin{array}{r}
\left|\left(\frac{d^{2}}{d z^{2}}+k^{2}\right) \Pi\left(R_{p}, z\right)\right| \ll\left|E_{z}^{(0)}\left(R_{p}, z\right)\right|, \\
z \in[-L / 2, L / 2], \quad p \in[1, N],
\end{array}
$$

holds true. Substituting (21) and (29) into (30), we arrive at the condition

$$
\begin{gathered}
\left|\sum_{q=1}^{N} R_{q} \sigma_{q} \int_{-L / 2}^{L / 2} E_{z}^{(0)}\left(R_{q}, z^{\prime}\right) G\left(z-z^{\prime}, R_{p}, R_{q}\right) d z^{\prime}\right| \\
\ll\left|\frac{c}{2} \int_{-L / 2}^{L / 2} E_{z}^{(0)}\left(R_{p}, z^{\prime}\right) \exp \left(i k\left|z-z^{\prime}\right|\right) d z^{\prime}\right|, \\
z \in[-L / 2, L / 2], \quad p \in[1, N],
\end{gathered}
$$

for the applicability of the first Born approximation. We found that condition (31) holds true for MWCNTs that are not too thick (i.e., $R_{N}$ is sufficiently small) in the frequency regime wherein the surface conductivities $\sigma_{p}$, $p \in[1, N]$, are mostly determined by interband transitions - the second term in (7). We estimate that MWCNTs with outermost radius $R_{N}<20 \mathrm{~nm}$ satisfy (29) at frequencies in the mid-infrared and the visible regimes.

Equation (29) contradicts the edge conditions (20). An analogous situation appears, for example, in the theory of diffraction by an aperture in an infinitesimally thin, perfectly conducting screen, wherein the exact solution must satisfy the Meixner condition ${ }^{53}$ on the aperture edge but an approximate solution based on the Huygens principle does not satisfy that condition. However, the error is strongly localized in the vicinity of the edge of the aperture and does not influence the scattered field in the far zone $\stackrel{57}{5}$ For our problem, (29) may be interpreted as a version of the Huygens principle for an MWCNT: the scattered field is generated by secondary current densities induced by the incident electric field on the surfaces of all shells. Therefore, the applicability of (29) is unphysical only in the vicinity of the edges $z= \pm L / 2$, but that should not affect the performance of the MWCNT as an antenna in the interband-transitions regime.

When the MWCNT is electrically thin in cross-section $\left(k R_{N} \ll 1\right)$, the further approximation

$$
E_{z}^{(0)}(\rho, z) \simeq E_{z}^{(0)}(0, z), \quad \rho \leq R_{N}
$$

is permissible. Then the substitution of (29) in (26) and (27) yields

$$
\begin{gathered}
P_{r}=\frac{\omega^{2}\left|\sigma_{T}\right|^{2}}{4 c^{3}} \int_{0}^{\pi} \sin ^{3} \theta\left|\int_{-L / 2}^{L / 2} e^{i k z \cos \theta} E_{z}^{(0)}(0, z) d z\right|^{2} d \theta \\
P_{t}=\frac{1}{2} \operatorname{Re}\left(\sigma_{T}\right) \int_{-L / 2}^{L / 2}\left|E_{z}^{(0)}(0, z)\right|^{2} d z
\end{gathered}
$$

where

$$
\sigma_{T}=\sum_{p=1}^{N}\left(2 \pi R_{p} \sigma_{p}\right)
$$

is the effective conductivity per unit length of an electrically thin MWCNT. The condition (31) leads to the inequality $P_{t} \gg P_{r}$; consequently,

$$
\eta \approx P_{r} / P_{t} \sim\left|\sigma_{T}\right|^{2} / \operatorname{Re}\left(\sigma_{T}\right) .
$$

As determined by (33) and (34), $P_{t}$ and $P_{r}$ are the same as for a thin-wire resistive antenna whose conductivity per unit length is equal to $\sigma_{T}, \underline{58}$ Thus, an electrically thin MWCNT which satisfies the condition (31) may be modeled as a thin homogenous cylinder with conductivity per unit length determined from (35).

\section{NUMERICAL RESULTS}

In order to present illustrative results, let us consider an MWCNT consisting of only zigzag shells. A large number of such MWCNTs are possible $\stackrel{1,2}{=}$ Moreover, after a suitable modification of the effective boundary conditions (4) and (5),, 52 the approach developed can be extended to MWCNTs comprising shells with arbitrary chirality vectors.

For definiteness, calculations were performed for two types of MWCNTs, hereafter referred to as type A and type M, shown in Fig. 1, The $p$-th shell in an MWCNT of type $\mathrm{A}$ is in the $(8 p+1,0)$ configuration; hence, two consecutive semiconducting shells are followed by a metallic shell. The $p$-th shell in an MWCNT of type M is in the $(9 p, 0)$ confuguration; hence, all $N$ shells are metallic. The radius of the $p$-th shell is given by

$$
R_{p}=\left\{\begin{array}{ll}
\sqrt{3}(8 p+1) b /(2 \pi), & \text { type } \mathrm{A} \\
9 \sqrt{3} p b /(2 \pi), & \text { type } \mathrm{M}
\end{array} .\right.
$$

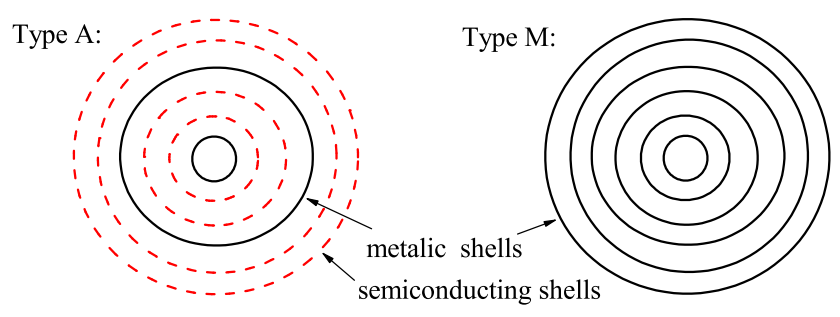

FIG. 1: Schematics of MWCNTs of types A and M. All shells in an MWCNT of type M are metallic. In contrast, an MWCNT of type A contains a metallic shell alternating with two semiconducting shells.

The frequency $f_{p}^{(1)}$ of the first interband transition for the $p$-th shell of the metallic type is determined by the 
energy difference between the lowest van Hove singularity of the conducting band and the highest van Hove singularity of the valence band. Thus, an analysis of the dispersion equation (10) for zigzag shells yields

$$
\begin{aligned}
f_{p}^{(1)} & =2 \mathcal{E}_{c}[2 \pi /(3 a), 3 p-1] /(2 \pi \hbar) \\
& \simeq v_{F} /\left(\pi R_{p}\right)
\end{aligned}
$$

where $v_{F}$ is the $\pi$-electron velocity at the Fermi level. The frequency of the first interband transition for the $p$ th shell of the semiconducting type is determined by the minimal band-gap energy as

$$
\begin{aligned}
f_{p}^{(1)} & =2 \mathcal{E}_{c}[2 \pi /(3 a), 3+[8(p-1) \pm 1] / 3] /(2 \pi \hbar) \\
& \simeq v_{F} /\left(3 \pi R_{p}\right),
\end{aligned}
$$

wherein the sign have to be chosen so that $[8(p-1) \pm 1] / 3$ is an integer. Thus, the frequency of the first interband transition is given by

$$
f_{p}^{(1)} \simeq \frac{v_{F}}{\mu_{p} \pi R_{p}}, \quad \mu_{p}=\left\{\begin{array}{l}
1, \text { metallic shell } \\
3, \text { semiconducting shell } .
\end{array}\right.
$$

The foregoing formula has been confirmed by direct numerical simulation $\underline{48}$

The frequency regime below the interband-transition regime for an MWCNT is dictated by the condition

$$
f<f_{e},
$$

where

$$
f_{e}=\stackrel{\min }{p}\left\{f_{p}^{(1)}\right\}
$$

while the condition

$$
f>(2 \pi \tau)^{-1}
$$

establishes the frequency regime for long-range guidedwave propagation. As a result, the frequency regime wherein long-range guided waves can produce geometric (antenna) resonances is as follows:

$$
(2 \pi \tau)^{-1} \lesssim f \lesssim f_{e}
$$

At frequencies in the regime $f \gtrsim f_{e}$, the interband transitions contribute strongly to the surface conductivity of each shell such that the real and the imaginary parts of this quantity are approximately equal in magnitude. Guided waves then get attenuated heavily. At frequencies in the regime $f \lesssim 1 /(2 \pi \tau)$, attenuation of guided waves is caused by fast electron relaxation in all shells.

\section{A. Guided waves in an infinitely long MWCNT}

Let us now examine guided-wave propagation at $f=$ $11.2 \mathrm{THz}$ in an infinitely long MWCNT of type A consisting of $N=13$ shells. The relaxation time $\tau$ is taken to be vanishingly small.
The shells numbered $p \in\{4,7,10,13\}$ are metallic. All interband transitions for these shells occur at frequencies exceeding $31 \mathrm{THz}$ and therefore do not contribute to the effective conductivity (per unit length) of the MWCNT. The imaginary part of the surface conductivity of a metallic shell is positive-valued and exceeds the real part in magnitude. Thus, the necessary condition for the long-range propagation of guided waves is satisfied.

The semiconducting shells labelled $p \in\{2,3,5,6\}$ have negligible surface conductivity, and therefore do not influence the scattering properties of the chosen MWCNT at $11.2 \mathrm{THz}$.

The surface conductivities of the semiconducting shells labelled $p \in\{8,9,11,12\}$ are dictated mainly by the corresponding first interband transitions occurring at $f_{p}^{(1)} \in\{36.5,32.5,26.7,24.4\} \mathrm{THz}$. At $f=11.2 \mathrm{THz}$. the imaginary parts of the surface conductivities of these shells are negative and several times smaller than the surface conductivities of adjacent metallic shells. Therefore, the surface current densities in these semiconducting shells are smaller and oppositely directed with respect to their counterparts in adjacent metallic shells. The real parts of the surface conductivities of these semiconducting shells are high enough to cause large ohmic losses.

We considered only the two eigenmodes of guided-wave propagation in the chosen MWCNT with the smallest retardation, labeled as $G W 1$ and $G W 2$. They correspond to the two roots of the dispersion equation (17), identified as $h_{1}$ and $h_{2}$, with the smallest real parts: $\operatorname{Re}\left(h_{2}\right)>\operatorname{Re}\left(h_{1}\right)$. Of all eigenmodes, these two will mostly influence the scattering properties of the finitelength MWCNT, as discussed in Sec. VB

The radial dependences of $E_{z}$ and $H_{\phi}$ inside the MWCNT of type A for guided waves $G W 1$ and $G W 2$ are shown in Fig. 2(a). Clearly, the axial component of the electric field is distributed over the entire cross section of the MWCNT. The azimuthal component of the magnetic field is discontinuous across each metallic shell, in accordance with the boundary condition (44). The degree of discontinuity decreases as the shell number $p$ increases, in compliance with the generally decreasing magnitudes of $J_{p}$ in Fig. 2(b). Outside the MWCNT, the radial distribution of the electric and magnetic fields is governed by the argument of the modified Bessel function $K_{0}\left(\sqrt{h^{2}-k^{2}} \rho\right)$, whereby we conclude that the electromagnetic field is highly localized to the MWCNT.

The axial surface current density $J_{p}, p \in[1, N]$, is shown in Fig. 2(b) for guided waves $G W 1$ and $G W 2$. In opposition to the radial distributions of the axial electric field, the magnitude of the current density is maximal on the innermost shell, and then strongly decreases with the increase of the shell number $p$. This behavior is in agreement with the $R^{-1}$-dependence of the surface conductivity of a metallic SWCNT of radius $R \underline{\underline{4}}$

Figure 2(c) shows the radial distribution of the axial 

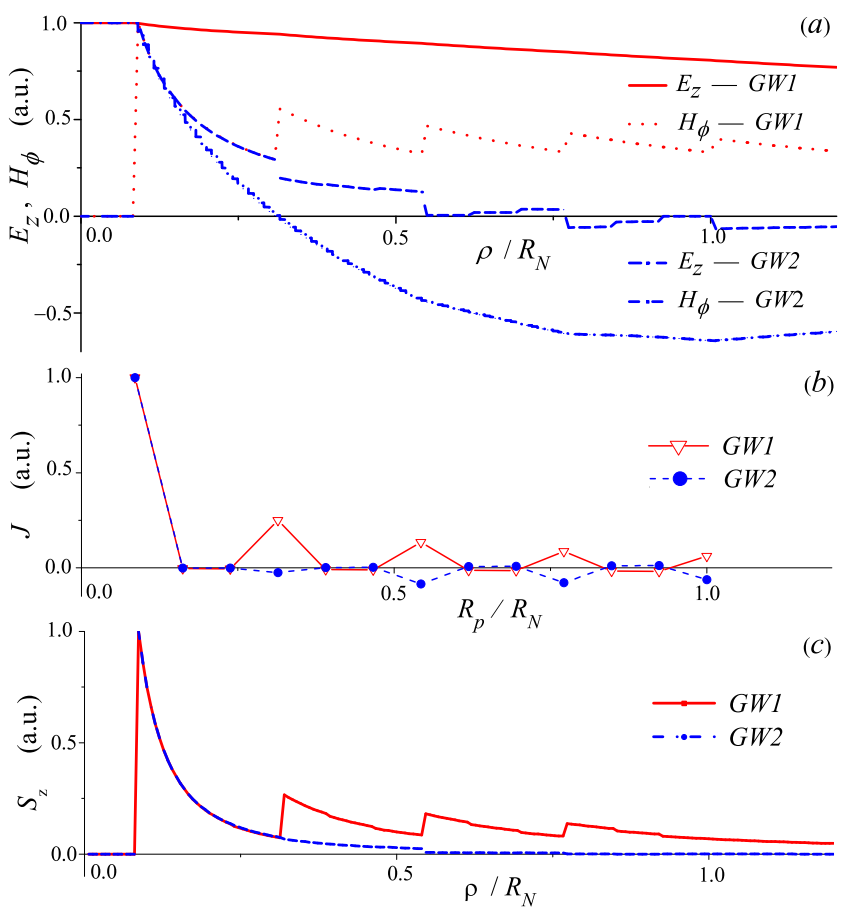

FIG. 2: The radial dependencies of (a) $E_{z}$ and $H_{\phi}$, (b) $J_{p}$ on the surfaces of the shells, and (c) the axial component of the time-averaged Poynting vector, for guided waves $G W 1$ and $G W 2$, in an MWCNT of type A with $N=13$ shells at $f=11.2 \mathrm{THz}$, in the limit $\tau \rightarrow \infty$. The discrete points in (b), corresponding to different shells, are joined together only to aid the eye.

component of the time-averaged Poynting vector

$$
S_{z}=\frac{c}{8 \pi} \operatorname{Re}\left(\frac{h}{k}\right)\left|H_{\phi}\right|^{2}
$$

for $G W 1$ and $G W 2$, inside the MWCNT and in the vicinity of its outermost shell. The energy-flux density for $G W 1$ is maximum near the surface of the innermost metallic shell and then slightly varies about some mean value between the $4^{\text {th }}$ and the $13^{\text {th }}$ shells. The energyflux density for $G W 2$ is mostly concentrated between the two innermost metallic shells and then decreases very rapidly as $\rho \rightarrow R_{N}$. Clearly then, the two innermost metallic shells are dominant contributors to the retardation of both $G W 1$ and $G W 2$.

The dependences of the real part of the slow-wave coefficient $\beta$ and the ratio $-\operatorname{Re}(\beta) / \operatorname{Im}(\beta)=\operatorname{Re}(h) / \operatorname{Im}(h)$ of the guided waves $G W 1$ and $G W 2$ on the number $N$ of shells in MWCNTs of types A and M are shown in Fig. 3. The retardation coefficient $\operatorname{Re}(\beta)$ for MWCNTs of type M is higher than for MWCNTs of type A of comparable $R_{N}$, which is a significant observation in relation to the geometric resonances of finite-length MWCNTs (Sec. VB).

For MWCNTs of type $\mathrm{M}, \operatorname{Re}(\beta)$ increases as $N$ does. As $N$ increases, the additional outmost shell has a lower
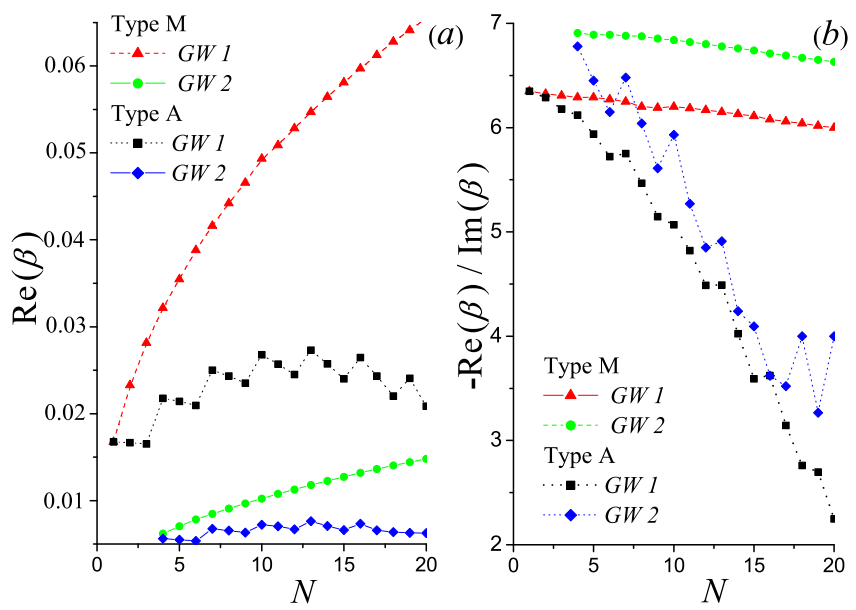

FIG. 3: Dependences of (a) $\operatorname{Re}(\beta)$ and $(\mathrm{b})-\operatorname{Im}(\beta) / \operatorname{Re}(\beta)$ on $N$ for guided waves $G W 1$ and $G W 2$ in MWCNTs of type A and $\mathrm{M}$, when $f=11.2 \mathrm{THz}$ and $\tau=10^{-13} \mathrm{~s}$. Discrete points are joined by lines to aid the eye.

influence on $\operatorname{Re}(\beta)$. The increase of $\operatorname{Re}(\beta)$ with increasing $N$ occurs until $R_{N}$ exceeds $v_{F} / \pi f$, per conditions (44) and (40).

Figure 3(a) shows that, for guided waves $G W 1$ and $G W 2$ in MWCNTs of type $\mathrm{A}, \operatorname{Re}(\beta)$

(i) increases with the addition of a metallic shell, but

(ii) decreases with the addition of a semiconducting shell.

For an MWCNT with small $N, \operatorname{Re}(\beta)$ is thus determined mostly by the metallic shells. In contrast, for MWCNTs with large $N$, both $\operatorname{Re}(\beta)$ and $-\operatorname{Re}(\beta) / \operatorname{Im}(\beta)$ are strongly affected by the semiconducting shells of large radius. This occurs because the semiconducting shells labelled $p \in\{11,12,14,15,17,18,20\}$ have their first interband transitions near the chosen frequency of $11.2 \mathrm{THz}$, and, consequently, the real and imaginary parts of their surface conductivities are large in magnitude. An increase in the number of such shells greatly diminishes the parameters $\operatorname{Re}(\beta)$ and $-\operatorname{Re}(\beta) / \operatorname{Im}(\beta)$ of $G W 1$ and $G W 2$ in the MWCNTs of type A. Thus, interband transitions suppress guided-wave propagation in MWCNTs.

\section{B. Scattering properties of a finite-length MWCNT}

Let us now move on to the scattering properties of finite-length MWCNTs in the terahertz and the farinfrared frequency regimes. Here we focus only on the case when the incident electric field is parallel to the $z$ axis, thereby permitting us to investigate electromagnetic effects caused by the axial surface conductivities of the shells. 


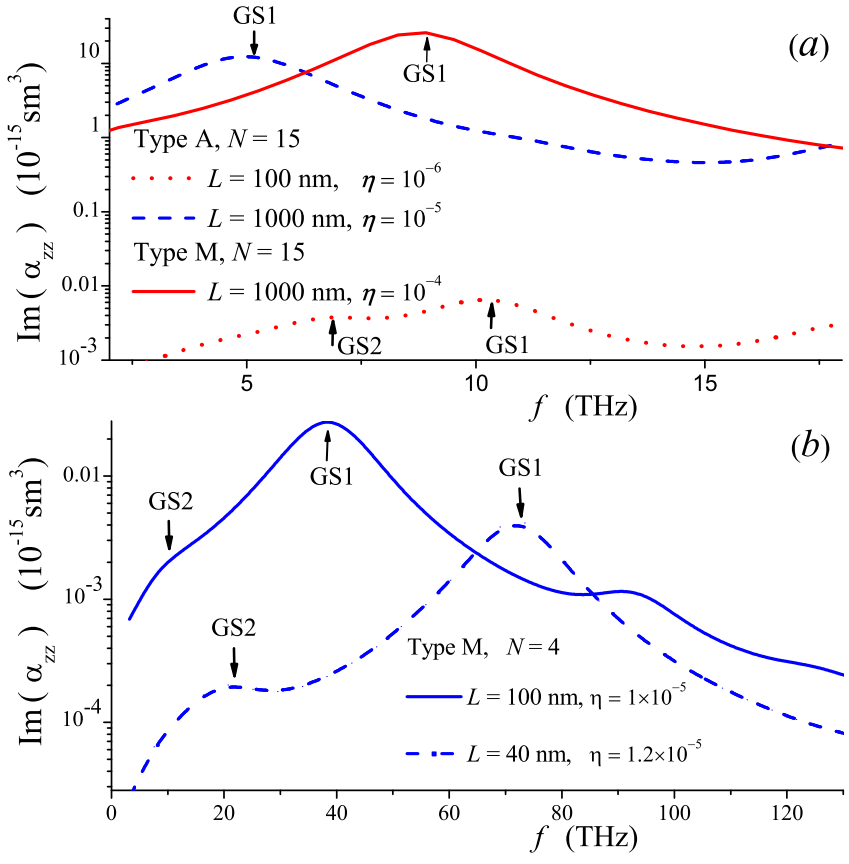

FIG. 4: Frequency-dependence of $\operatorname{Im}\left(\alpha_{z z}\right)$ of MWCNTs of type A and $\mathrm{M}$ for different lengths $L$ and number of shells $N$. The labels $G S 1$ and $G S 2$ denote the first geometric resonance - $s=1$ in (46) — of the guided waves $G W 1$ and $G W 2$, respectively. (a) $\tau=10^{-13} \mathrm{~s}$, (b) $\tau=2 \times 10^{-14} \mathrm{~s}$.

Figure 4(a) illustrates the frequency dependence of the imaginary part of the polarizability scalar $\alpha_{z z}$ in the long-wavelength regime $(k L \ll 1)$ for different lengths $L$ and shell numbers $N$ in MWCNTs of type A and M. The labels $G S 1$ and $G S 2$ in this figure denote the first geometric resonance of the guided waves $G W 1$ and $G W 2$, respectively. The geometric resonances occur at frequencies 16.33

$$
f_{s}=s\left(\frac{c}{2 L}\right) \operatorname{Re}(\beta), \quad s \in\{1,3,5, \ldots\} .
$$

As we concluded from Fig. 3(a), the retardation coefficient $\operatorname{Re}(\beta)$ for MWCNTs of type M is higher than for MWCNTs of type A of comparable $R_{N}$. Therefore the first geometric resonance $(s=1)$ for the MWCNTs of type $\mathrm{M}$ appears in Fig 4 (a) at a higher frequency than for MWCNTs of type A, both of the same length $L$.

The resonance frequencies for $G W 1$ and $G W 2$ depend on $L$ nonlinearly. As an example, in Fig. 廿(a) the resonance frequency of the MWCNT of type A increases by a factor of about 2 (from 5 to $\sim 10 \mathrm{THz}$ ) while the length $L$ decreases by a factor of 10 (from 1000 to $100 \mathrm{~nm}$ ). That is reflected in (46) by the strong dependence of $\operatorname{Re}(\beta)$ on $f$, when $f$ is close to $f_{e}$. Accordingly, the dependence of the frequency of the geometric resonance on $L$ is not explicit.

The first resonance of the MWCNT of type A and length $L=100 \mathrm{~nm}$ at $\sim 10-\mathrm{THz}$ frequency is not strong, because condition (44) is not satisfied for this MWCNT, and the guided wave is strongly attenuated. We conclude that the resonance of an MWCNT antenna of type A or $\mathrm{M}$ near a given frequency are the most pronounced, if both (44) and (46) are satisfied.

Figure 4(a) contains the value of the antenna efficiency $\eta$ at the first geometric resonance for all MWCNTs considered. This antenna efficiency depends both on the type and the length of the MWCNT. The antenna efficiency of an MWCNT is several time larger than that of an SWCNT of the same length. Our calculations make us conclude that the restrictions on the MWCNT dimensions given by (44) and (46) do not permit an increase in $\eta$ at the first geometric resonance. In contrast, the antenna efficiency of an almost circular bundle of closely packed SWCNTs can be increased up to unity, by increasing of number of metallic SWCNTs in the bundle $:^{33}$

When conduction in an MWCNT is very diffuse, $\stackrel{59}{t}$ the relaxation time $\tau$ is close to that for graphite $\left(2 \times 10^{-14} \mathrm{~s}\right)$. Then the conditions (44) and (46) can be fulfilled only for MWCNTs of type M, and that too with $R_{N} \lesssim 2.5 \mathrm{~nm}$ and $L \lesssim 200 \mathrm{~nm}$. Figure 4 (b) shows the frequency dependence of $\operatorname{Im}\left(\alpha_{z z}\right)$ of an MWCNT of type M with $N=4$ for $\tau=2 \times 10^{-14} \mathrm{~s}$. The first geometric resonances of MWCNTs of lengths $L=100 \mathrm{~nm}$ and $L=40 \mathrm{~nm}$ appear in the far-infrared $(f=38 \mathrm{THz})$ and the mid-infrared $(f=72 \mathrm{THz})$ regimes, respectively. Thus, antenna resonances are pronounced and can be experimentally observed for short MWCNTs with several shells only in the far-infrared and mid-infrared regimes.

\section{MWCNT properties in the interband-transitions regime}

Whereas Secs. $\mathrm{VA}$ and $\mathrm{VB}$ address the frequency regime $f \in\left(1 / 2 \pi \tau, f_{e}\right)$ wherein interband transitions are not possible, we now proceed to the interband-transitions regime of Sec.IVB, wherein the electromagnetic response properties of MWCNTs are dominated by the interband transitions. This regime, delineated by the condition $f>f_{e}$, can very well lie in the visible part of the electromagnetic spectrum.

Wang et al. have published the only known report on the experimental observation of antenna resonances of MWCNTs in the visible regime $\stackrel{13}{\underline{1 n}}$ In their experiment, interference patterns of light scattered from an array of finite-length MWCNTs with $R_{N} \approx 25 \mathrm{~nm}$ were observed. Wang et al. attributed the observed phenomenon to a length-matching antenna effect, which requires the spatial variations of the induced current density to satisfy the edge conditions; furthermore, they assumed that a thick MWCNT has the same scattering response in the far zone as a perfect conducting cylinder with the same cross-sectional radius and length. However, Hao and Hanson ${ }^{32}$ subsequently showed that the model of an MWCNT as a single cylindrical shell with typical surface conductivity given, e.g., by (7), can not describe the experimental observation recorded in Ref. 13. The reason 
is the low surface conductivity of the MWCNT shells, as given by (7). In the model of Ref. 32, however, the intershell electromagnetic coupling in the MWCNT was ignored, which is inappropriate if $R_{N} \gtrsim 20 \mathrm{~nm}$, as we pointed out in Sec. IVB.

The lacuna in the model of Ref. 32 can be removed by implementing the integral-equation technique of Sec. IVA Therefore, we decided to examine the scattering properties of finite-length MWCNTs of radius $R_{N} \simeq 25 \mathrm{~nm}$ and $R_{N} \simeq 50 \mathrm{~nm}$ in the visible regime, taking the intershell electromagnetic coupling into account (but ignoring the intershell electron tunneling). As we discuss later in this subsection, our analysis confirms the absence of the length-matching antenna effect in the near-infrared and the visible regimes.

Let us first analyze the effective conductivity per unit length of an electrically thin MWCNT, as estimated by (35). Figure 5 (a) presents the real part of $\sigma_{T}$ as a function of $N$ for MWCNTs in the near-infrared and the visible regimes. This figure shows that, for SWCNTs $(N=1)$ and MWCNTs with $N \in[2,4], \sigma_{T}$ has strong resonances. The resonances weaken as $N$ increases and practically disappear for $N>10$. This trend can be explained in the following way. In the near-infrared and the visible regimes, the surface conductivity $\sigma_{p}$ of the $p$-th shell, obtained from (7), has many resonances corresponding to van Hove singularities. As the resonances of different shells overlap, the weighted summation of the surface conductivities of all shells in (35) ensures that $\sigma_{T}$ does not evince resonant behavior in the near-infrared and the visible regimes.

The conductivity per unit length of an isolated shell of radius $R_{p} \gtrsim 5 \mathrm{~nm}$ also has a large number of closely spaced resonances so that, instead of discrete lines, a band appears in its spectrum. As the surface conductivities of all MWCNT shells have a plasmon resonance $\underline{\underline{60}}$ in the ultraviolet regime at the free-space wavelength $\lambda_{p l}=(c \pi \hbar) / \gamma_{0}, \sigma_{T}$ for any MWCNT also has a resonance at this wavelength.

The real and imaginary parts of the effective per-unitlength conductivity $\sigma_{T}$ for an MWCNT of type M with $N=29$ shells (i.e., $R_{N}=10.22 \mathrm{~nm}$ ) are presented in Fig. 5(b). As this figure demonstrates, the real part of $\sigma_{T}$ increases as the frequency increases and has a maximum near $f=1304 \mathrm{THz}$ (i.e., $\lambda=230 \mathrm{~nm}$ ), which is not a geometric-resonance frequency but, instead, is a plasmon-resonance frequency $\underline{\underline{60}}$ In the near-infrared and the visible regimes, the condition $\operatorname{Re}\left(\sigma_{T}\right) \gg \operatorname{Im}\left(\sigma_{T}\right)$ holds; therefore, the chosen MWCNT cannot support surface-wave propagation, which occurs for a metallic wire with $\operatorname{Re}(\sigma) \ll \operatorname{Im}(\sigma)$ according to Ref. 61. We also found that the electric field exciting a particular shell differs very slightly from the electric field incident on the MWCNT, when $R_{N} \lesssim 20 \mathrm{~nm}$ and the frequency lies in either the near-infrared or the visible regimes, thereby implying that the electromagnetic coupling between the shells is slight. The frequency dependence of the scattering power $P_{t}$ for such an MWCNT is the same as of
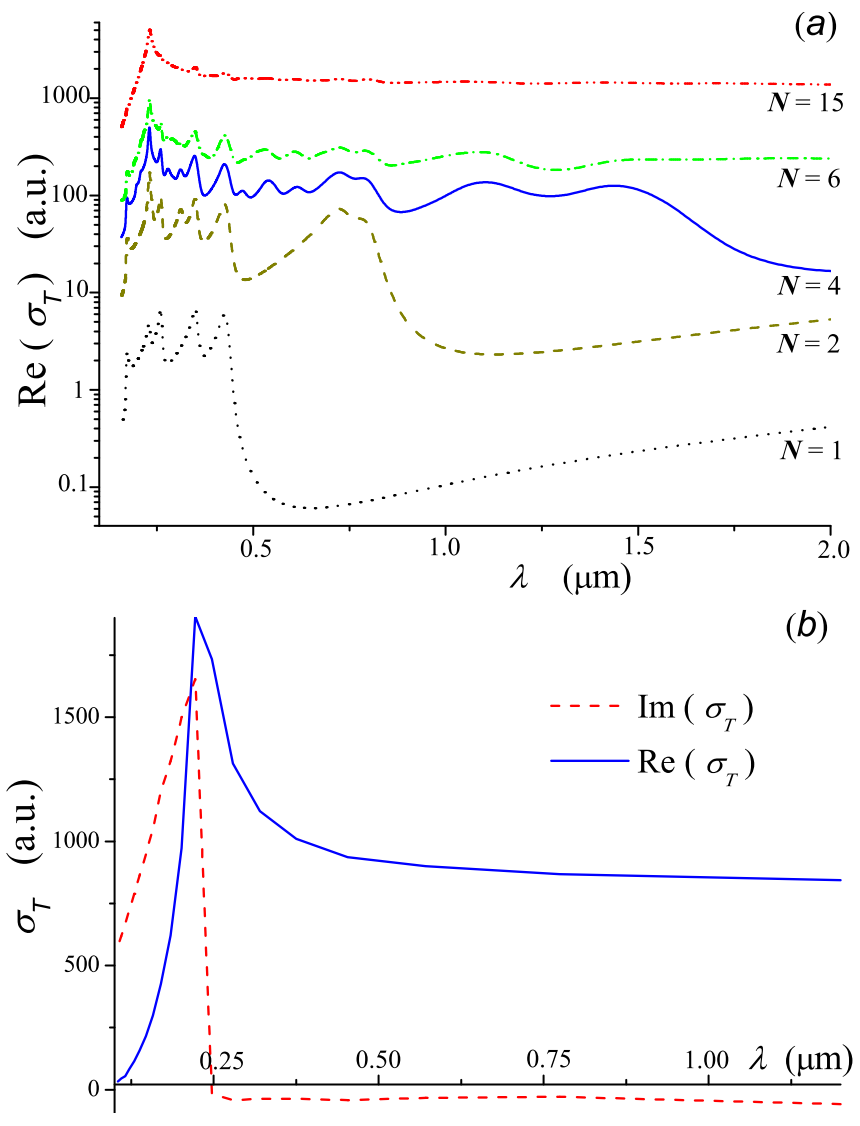

FIG. 5: (a) Real part of the effective conductivity per unit length of an MWCNT of type M in the near-infrared and the visible regimes. The number $N$ is variable, and $\tau=2 \times$ $10^{-14} \mathrm{~s}$. Note that $R_{15}=5.284 \mathrm{~nm}$. (b) Real and imaginary parts of $\sigma_{T}$ of an MWCNT of type M with $N=29$ shells in the near-infrared and the visible regimes.

$\left|\sigma_{T}(\omega)\right|^{2}$, according to (33).

Let us now carry on to electrically thick MWCNTs (with $R_{N} \simeq 25 \mathrm{~nm}$ or $50 \mathrm{~nm}$ ). For calculation of the electric current densities in their shells in the near-infrared and the visible regimes we used (23), with $E_{z}^{(0)}(\rho, z)$ assumed to be independent of $\rho \in\left[0, R_{N}\right]$. Such an approximation is sufficient to ascertain whether geometric resonances of azimuthally symmetrical modes exist in thick MWCNTs or not. Of course, $\sigma_{T}$ cannot be defined for electrically thick MWCNTs.

In the near-infrared and visible regimes, the surface conductivity of a shell of large radius $(R \gtrsim 5 \mathrm{~nm})$, in accordance with (7), does not depend on that whether shell is metallic or semiconducting. Therefore, though the plots in Figs. 6] were made for thick MWCNTs of type M, all results presented therein are qualitatively true for thick MWCNTs of type A also.

In order to compare the electromagnetic responses of the chosen MWCNTs and a perfectly conducting rod in the near-infrared and the visible regimes, we need to calculate the scattered power $P_{r}$ when the incident electric 


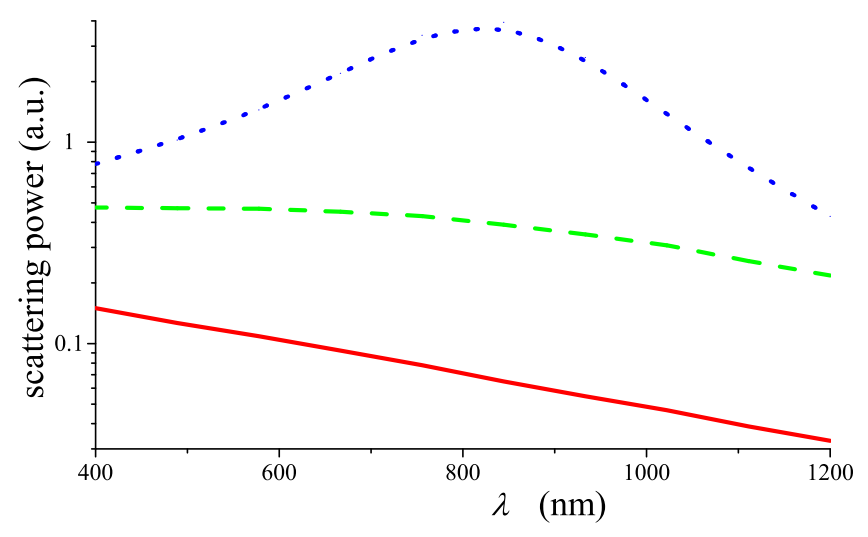

FIG. 6: Scattered power $P_{r}$ versus free-space wavelength $\lambda$ for MWCNTs with $N=70$ (solid line) and $N=140$ shells (dashed line) and length $L=350 \mathrm{~nm}$. The scattered power for a perfectly conducting rod (dotted line) of cross-sectional radius $25 \mathrm{~nm}$ and length $350 \mathrm{~nm}$ was calculated by solving (23) with $N=1$ and $\sigma_{1} \rightarrow \infty$.

field is oriented parallel to the $z$ axis. When we calculated the scattered power $P_{r}$ for MWCNTs of type M, length $L=350 \mathrm{~nm}$, and $N=70\left(R_{N}=24.66 \mathrm{~nm}\right)$ or $N=140\left(R_{N}=49.32 \mathrm{~nm}\right)$, resonances did not show up for $f \in[250,750] \mathrm{THz}$ (i.e., $\lambda \in[400,1200] \mathrm{nm}$ ) in Fig. 6. For comparison, the scattered power for a perfectly conducting rod of cross-sectional radius $25 \mathrm{~nm}$ and length $350 \mathrm{~nm}$ is also shown in Fig. 6. This nanorod antenna, in contrast to the MWCNTs, has a set of resonances determined by the condition

$$
L=\kappa s \lambda / 2, \quad s \in\{1,2,3, \ldots .\},
$$

where $\kappa$ is a correction factor that slightly exceeds unity and is a function of the ratio of the length and the wavelength as well as of the ratio of cross-sectional radius and the length .51 For the chosen nanorod antenna, the first resonance $(s=1)$ is characterized by $\kappa=1.15$ and appears at $f=370 \mathrm{THz}$ (i.e., $\lambda=810$ ) nm, which is confirmed by the dotted line Fig. 6 .

The absence of antenna (geometric) resonances of the chosen MWCNTs in the visible regime can be explained by the strong dissipation of electromagnetic energy in MWCNT shells and the small electromagnetic coupling between the shells, as discussed in Sec. IVB. Therefore, even an MWCNT with $R_{N} \simeq 50 \mathrm{~nm}$ and comprising 140 shells can not support guided-wave propagation and, consequently, can not display the length-matching antenna effect. The same conclusion is also true in the visible regime for SWCNTs 16 and planar arrays of finite-length SWCNTs $\stackrel{32}{=}$ Let us remark that a hypothetic multishell conductive structure with $N=70$ and $R_{N} \simeq 25 \mathrm{~nm}$ can have an antenna resonance corresponding to $s=1$ in (47), provided the surface conductivity of every shell is five times that given by (7); but such a structure has not been practically realized as yet.

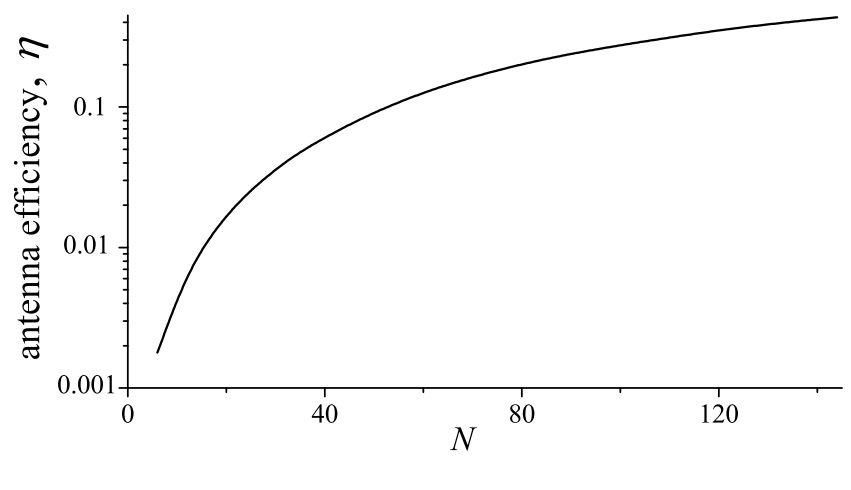

FIG. 7: Dependence of the antenna efficiency $\eta$ on the number $N$ of shells in an MWCNT of type M and length $L=350 \mathrm{~nm}$ at frequency $f=600 \mathrm{THz}$ (visible regime).

Our approach, which is enhanced with respect to that of Hao and Hansen, 32 can not explain the MWCNT antenna resonance reported by Wang et al $\underline{13}$ Additional experiments are necessary to resolve the contradiction between interpretation of measurements proposed by Wang et al. and the theoretical model of MWCNTs developed here and based on Ref. 4 .

The dependence of the antenna efficiency $\eta$ in the visible regime on the number $N$ of shells in the MWCNT is illustrated in Fig. 7 The antenna efficiency increases with the number of shells and tends to unity for thick MWCNTs; indeed, $f=600 \mathrm{THz}$, we calculated $\eta=0.17$ for $N=70$, but $\eta=0.44$ for $N=140$.

\section{Surface-plasmon-wave propagation in an MWCNT with a gold core}

These days, thin metallic (gold, silver, and aluminium) wires of finite length are considered to be promising for application as optical nanoantennas 54,62 However, the fabrication of long, high-quality, thin, single-crystal wires of cross-sectional radius less than $5 \mathrm{~nm}$ and with perfect cylindrical form (i.e., without breaks, bends, deformations, etc.) is a difficult technological problem. Recently, CNTs have been used as templates in order to promote the formation of high-quality single-crystal wires coated by perfect graphene cylinders ${ }^{63}$ This is an exciting possibility for a composite nanoantenna comprising a solid metallic core covered by concentric carbon shells. Clearly, such a structure is neither a metallic cylinder nor an isolated MWCNT.

Surface-plasmon waves in the infrared and the visible regimes can propagate along a metal wire ${ }^{61}$ Surely, guided-wave propagation would be affected if the metallic wire were to be enclosed in an ensemble of concentric carbon shells. The theoretical approach of Sec. III can be applied to study the phenomenon of surface-plasmon waves as follows.

Suppose that the metal core is of cross-sectional ra- 

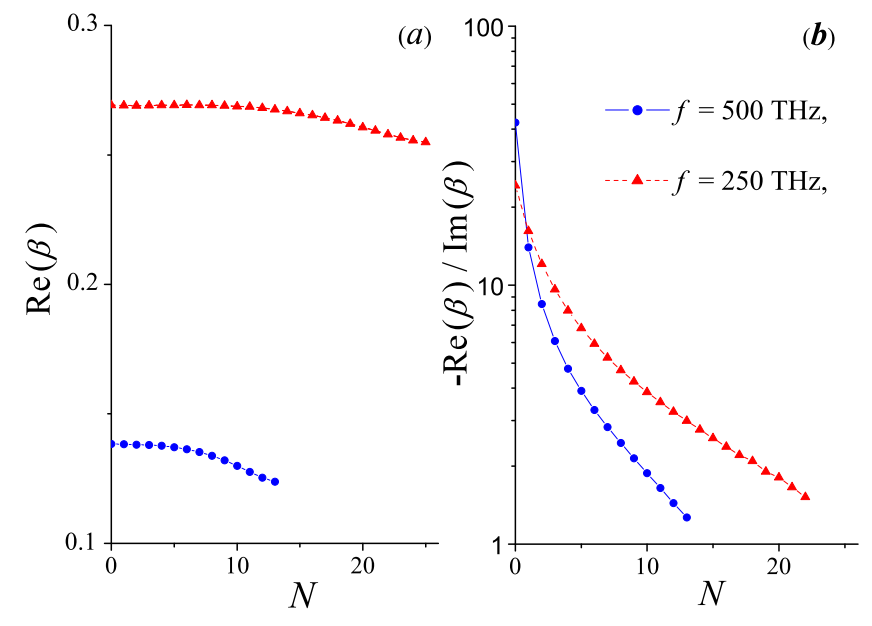

FIG. 8: Dependences of (a) $\operatorname{Re}(\beta)$ and (b) the ratio $-\operatorname{Re}(\beta) / \operatorname{Im}(\beta)$ on the number $N$ of carbon shells for a surfaceplasmon wave in an MWCNT with a gold core. The MWCNT is of type $\mathrm{M}$, and its $p$-th shell has a $(90+9 p, 0)$ zigzag configuration and radius $R_{p}=\sqrt{3}(90+9 p) b /(2 \pi), p \in[1, N]$. The gold core has a cross-sectional radius $R_{0}=3.5 \mathrm{~nm}$. Data for an isolated metal wire of cross-sectional radius $R_{0}$ is the same as for $N=0$. Discrete points are joined by lines to aid the eye.

dius $R_{0}<R_{1}$, the radius of the innermost shell of an MWCNT. If $R_{0}$ is much less than the skin depth of the bulk metal and $k R_{0} \ll 1$, then the metal core can be modeled as a solid cylinder with surface conductivity $\sigma_{0}=\sigma_{m e t} R_{0} / 2 \stackrel{64}{\underline{w}}$ where $\sigma_{m e t}$ is the bulk volumetric conductivity of the metal. Also, $R_{0}$ has to be higher than a critical value $R_{c r}$, which corresponds to the crystalline-noncrystalline transition in metals and separates the quasi-bulk behavior $\left(R_{0}>R_{c r}\right)$ from the quasimolecular behavior $\left(R_{0}<R_{c r}\right)$ of a nanowire. Then, (44) -(6) also hold at $\rho=R_{0}$ with $\sigma_{p} \rightarrow \sigma_{0}$, and the procedures of Sec. III are applicable.

We considered the propagation of surface-plasmon waves in an MWCNT with a gold core. The bulk volumetric conductivity $\sigma_{m e t}$ of gold was taken to follow the Drude model with parameters given in Ref. 65. The skin depth of gold in the visible regime is higher than $30 \mathrm{~nm}$. The value $R_{c r}=1.5 \mathrm{~nm}$ for gold was obtained with firstprinciples calculations in Ref. 66. So we assumed that $1.5 \mathrm{~nm}<R_{0} \ll 30 \mathrm{~nm}$.

Figure 8 presents the dependences of $\operatorname{Re}(\beta)$ and $-\operatorname{Re}(\beta) / \operatorname{Im}(\beta)$ of a surface-plasmon wave on the number $N$ of shells in MWCNT of type M with a gold core of cross-sectional radius $R_{0}=3.5 \mathrm{~nm}$. The $p$-th shell of the MWCNT has a $(90+9 p, 0)$ zigzag configuration and radius $R_{p}=\sqrt{3}(90+9 p) b /(2 \pi), p \in[1, N]$. In the near-infrared $(f=250 \mathrm{THz})$ and the visible $(f=500$ $\mathrm{THz}$ ) regimes, Fig. 8(a) shows that the retardation coefficient $\operatorname{Re}(\beta)$ depends only slightly on $N$. According to Fig. 8(b), the value of $-\operatorname{Re}(\beta) / \operatorname{Im}(\beta)$ is less than for an isolated metal wire $(N=0)$ and significantly decreases as $N$ increases.

These trends can be explained in the following manner. The guided wave propagates partly inside the MWCNT and partly inside the gold core. The dissipation in the MWCNT shells is high because the real and the imaginary parts of the surface conductivities of the shells in the interband regime are of comparable magnitudes. This dissipation increases with frequency, in accordance with the frequency dependence of the effective per-unit-length conductivity of the MWCNT presented in Fig. 5(b). Because of the weak electromagnetic coupling of CNT shells, the shells strongly absorb electromagnetic energy independently on each other - which explains the strong dependence of $-\operatorname{Re}(\beta) / \operatorname{Im}(\beta)$ on $N$. Furthermore, the weak coupling changes the radial electromagnetic field distribution of the surface-plasmon wave only slightly as $N$ increases, which explains the weak dependence of $\operatorname{Re}(\beta)$ on $N$.

The decrease of the magnitude of $-\operatorname{Re}(\beta) / \operatorname{Im}(\beta)$ with increasing $N$ increasing implies the enhancement of $P_{t}$, the power lost to ohmic dissipation, and consequently the decreasing of the antenna efficiency $\eta$ of an MWCNT with a metal core. However, if $N$ is not too large $(N=2$ or 3 ) some worsening of antenna properties may be justified by other advantages that the metal-core MWCNT may confer.

\section{DISCUSSION}

Thus, an MWCNT can operate as an antenna in two different regimes. The first is the Drude-conductivity regime, wherein the motion of conduction-band electrons is responsible for radiation properties. This regime has a distinct analogy with a classical radio-frequency wire antenna, as is clear from Sec. IVB and VB. The second regime is the interband-transitions regime, wherein quantum transitions of electrons between different energy states occur. This regime was considered in Sec. VC and does not have a classical analogy. The frequency $f_{e}$ separates the Drude-conductivity regime $f \in\left(1 / 2 \pi \tau, f_{e}\right)$ from the interband-transitions regime $f>f_{e}$.

Guided-wave propagation and geometric resonances of the guided waves are typical for macroscopic wire antennas. The guided waves have a quasi-transverseelectromagnetic structure and are characterized by low retardation and low attenuation $\underline{\underline{51}}$ The existence of guided (surface) waves and geometric resonances also is typical for nanowire antennas in the Drude-conductivity regime., 16,62 But the guided wave has strong retardation and high attenuation, so that the frequency of a geometric resonance is not connected to the free-space wavelength but to a shorter effective wavelength that depends on the material properties. $\frac{54}{.}$ This general rule is also true for MWCNT antennas: Fig. 3 shows that guided waves have strong retardation and high attenuation, and Fig. [4 presents geometric resonances at $\lambda \ll L$ demonstrating thereby the effective wavelength to be shorter than free- 
space wavelength.

Calculated data presented in Fig. 3)(a) indicate that the retardation coefficient increases when the number of shells increases. Furthermore, the retardation coefficient is the highest for the $G W 1$ mode in MWCNTs of type M. That implies that a MWCNT with only metallic shells and operating in the $G W 1$ mode offers attractive prospects for high antenna efficiencies in the terahertz regime.

The frequency $f_{e}$, separating the Drude-conductivity regime from the interband-transitions regime, depends on the detailed electronic and geometric attributes of the MWCNT. According to (40), $f_{e}$ decreases as $R_{N}$ increases. As examples, for an MWCNT of type A, (i) $R_{N}=10 \mathrm{~nm}$ and $f_{e}=9.3 \mathrm{THz}$ when $N=32$, but (ii) $R_{N}=1.9 \mathrm{~nm}$ and $f_{e}=48 \mathrm{THz}$ when $N=6$. In the interband-transitions regime $f>f_{e}$, guided-wave propagation and geometric resonances are absent for both SWCNTs and MWCNTs, which cardinally distinguishes this regime from the Drude-conductivity regime.

As shown experimentally, ${ }^{67}$ the absorption and the scattering characteristics of an electrically thick MWCNT in the regime of optical transitions depend only slightly on the frequency. This conclusion, also borne out by the data in Fig. 6, may be seem to be unexpected. In fact optical transitions are resonance processes and the surface conductivities of SWCNTs and MWCNT shells have resonances corresponding to the van Hove singularities. But we found that the antenna parameters of electrically thin MWCNTs are determined by the effective parameter $\sigma_{T}$ defined in (35). The overlapping of a large number of resonances of the surface conductivities of the different shells leads to a smooth frequency dependence of $\sigma_{T}$. Such an effect is analogous to inhomogeneous broadening in an ensemble of all different harmonic oscillators.

Thus, within the framework of our model, it is impossible to interpret the observation scattering resonances of random arrays of MWCNTs with average outermost radius $25 \mathrm{~nm}$ reported in Ref. 13. The same conclusion emerged from the model of Hao and Hansen 32 Further experiments are required.

Antennas are objects that transform a near-field into a far-field and vice versa. The morphology of the near field of a nanoantenna possess a nanoscale, and is therefore determined by quantum size effects. Therefore the coupling of a nanoantenna with its near field is stronger than that of a macroscopic antenna with its near field; as a result, the transformation of the near field to the far field by a nanoantenna is more difficult, and the antenna efficiency of a thin-nanowire antenna is low. This property is inherent to different types of nanoantennas: SWCNTs ${ }^{14,15}$, bundle of $\mathrm{SWCNTs}^{33}$, metallic nanorods 19 , and MWCNTs as in Fig. 4. The antenna efficiency can be enhanced by increasing the number of shells in an MWCNT (Fig. (7), the number of SWCNTs in a bundle (Fig. 7 in Ref. 33), and the cross-sectional radius of a metallic rod antenna (Fig. 1 in Ref. 19). We can thus conclude that the small value of antenna efficiency is the fundamental physical characteristic of nanoantennas. Nevertheless, its antenna efficiency is tunable over a wide range.

In order to achieve antenna efficiency close to unity, it is necessary to strongly suppress the influence of quantum size effects by ensuring that the cross-sectional radius is high. Note that, since quantum size effects are not pronounced in gold nanowires of cross-sectional radius of several tens of nanometers, such nanowire-based antennas are expected to possess properties analogous to macroscopic antennas.

The electromagnetic properties of MWCNTs have only a slight frequency-dependence in the interbandtransitions regime, per Fig. 6. Thus, and MWCNT can be considered to be a nanoantenna with sufficiently high $\eta$ ( $\approx 0.1$ in Fig. 7) and a wide operating-frequency range in the visible regime. Such nanoantennas have properties similar to those of electrically small but macroscopic antennas in microwave regimes (e.g., short non-resonant dipoles) .51

\section{CONCLUDING REMARKS}

To conclude, we modeled the shells of an MWCNT as impedance sheets with axially directed surface conductivity, ignored intershell tunneling of electrons but incorporated intershell coupling, in an integral-equation approach. Calculated data indicate that in a low-frequency regime called the Drude-conductivity regime, wherein optical interband transitions do not occur, guided waves can propagate with low attenuation in an MWCNT which has metallic shells. In the same frequency regime, the axial polarizability of a finite-length MWCNT has a resonant behavior due to the antenna-length matching effect. However, the shells with surface conductivity due to interband transitions suppress guided-wave propagation. Due of the high dissipation in such shells, MWCNTs with outermost radius $\approx 25 \mathrm{~nm}$ can not possess resonant properties in the visible regime. Analysis of surface-plasmonwave propagation in a MWCNT with a gold core shows that, in the near-infrared and the visible regimes, the shells behave effectively as lossy dielectric materials and suppress surface-wave propagation along the gold core.

The following conclusions regarding the operation of MWCNTs as nanoantennas emerged from our work:

(i) The antenna efficiency $\eta$ of an MWCNT exceeds that of an SWCNT but is less than that of an almost circular bundle of closely packed, metallic SWCNTs, provided that all three objects are of roughly the same outermost radius. Therefore, SWCNT-bundles are the most promising candidates for terahertz and mid-infrared antennas.

(ii) An MWCNT with at least 4 shells is recommended for application as a nanoantenna with a wide operating-frequency range in the visible regime. 
(iii) Filling the core of an MWCNT with a metal makes the MWCNT attractive as a nanoantenna, provided that the number of shells does not exceed 3 .

The model developed in this paper can be applied for an MWCNT with achiral shells and negligibly small intershell tunneling. Generalization of the boundary condition (4)-(7) is needed to take the chirality of shells and intershell tunneling into account.

\section{Acknowledgments}

This research was partially supported by INTAS under projects 05-1000008-7801 and 06-1000013-9225, Interna- tional Bureau BMBF (Germany) under project BLR 08/001, and the Belarus Republican Foundation for Fundamental Research and CNRS (France) under project F07F-013. MVS thanks the World Federation of Scientists for a fellowship. AL acknowledges the Charles Godfrey Binder Professorship Endowment at the Pennsylvania State University for partial support.
1 M. S. Dresselhaus, G. Dresselhaus, and Ph. Avouris, Carbon Nanotubes (Springer, Berlin, Germany, 2001).

2 S. Reich, C. Thomsen, and J. Maultzsch, Carbon Nanotubes. Basic Concepts and Physical Properties (WileyVCH, Berlin, Germany, 2004).

3 M. L. Schipper, N. Nakayama-Rachford, C. R. Davis, N. W. S. Kam, P. Chu, Z. Liu, X. Sun, H. Dai, and S. S. Gambhir, Nature Nanotechnol. 3, 216 (2008).

${ }^{4}$ G. Ya. Slepyan, S. A. Maksimenko, A. Lakhtakia, O. Yevtushenko, and A. V. Gusakov, Phys. Rev. B 60, 17136 (1999).

5 S. A. Maksimenko and G. Ya. Slepyan, in Electromagnetic Fields in Unconventional Materials and Structures (O. N. Singh and A. Lakhtakia, eds.), (Wiley, New York, NY, USA, 2000), pp. 217-255.

6 S. A. Maksimenko and G. Ya. Slepyan, Nanoelectromagnetics of low-dimensional structures, in Nanometer Structures: Theory, Modeling, and Simulation (A. Lakhtakia, ed.), (SPIE Press, Bellingham, WA, USA, 2004), pp. 145206.

7 M. J. Hagmann, IEEE Trans. Nanotechnol. 4, 289 (2005).

8 J. Rybczynski, K. Kempa, A. Herczynski, Y. Wang, M. J. Naughton, Z. F. Ren, Z. P. Huang, D. Cai, and M. Giersig, Appl. Phys. Lett. 90021104 (2007).

9 A. Raychowdhury and K. Roy, IEEE Trans. CAD Integrat. Circ. Syst. 25, 58 (2006).

10 A. G. Chiariello and G. Miano, COMPEL: Int. J. Comp. Math. Electrical Electron. Engg. 26571 (2007).

11 A. Maffucci, G. Miano, and F. Villone, Int. J. Circ. Theory Appl. 36, 31 (2008).

12 H. Li, W.-Y. Yin, K. Banerjee, and J.-F. Mao, IEEE Trans. Electron Devices 55, 1328 (2008).

13 Y. Wang, K. Kempa, B. Kimball, J. B. Carlson, G. Benham, W. Z. Li, T. Kempa, J. Rybczynski, A. Herczynski, and Z. F. Ren, Appl. Phys. Lett. 85, 2607 (2004).

14 G. W. Hanson, IEEE Trans. Antennas Propagat. 53, 3426 (2005).

15 P. J. Burke, S. Li, and Z. Yu, IEEE Trans. Nanotechnol. 5, 314 (2006).

16 G. Ya. Slepyan, M. V. Shuba, S. A. Maksimenko, and A. Lakhtakia, Phys. Rev. B 73, 195416 (2006).

17 K. Kempa, J. Rybczynski, Z. Huang, K. Gregorczyk, A.
Vidan, B. Kimball, J. Carlson, G. Benham, Y. Wang, A. Herczynski, and Z. F. Ren, Adv. Mater. 19, 421 (2007).

18 Y. Wang, Q. Wu, W. Shi, X. He, X. Sun, and T. Gui, Int. J. Infrared Millim. Waves 29, 35 (2008).

19 G. W. Hanson, IEEE Antennas Propagat. Mag. in press, June (2008).

20 S. A. Maksimenko, G. Ya. Slepyan, A. M. Nemilentsau, and M. V. Shuba, Physica E 40, 2360 (2008).

${ }^{21}$ C. Rutherglen and P. Burke, Nano Lett. 7, 3296 (2007).

22 K. Jensen, I. Weldon, H. Garcia, and A. Zettl, Nano Lett. 7, 3508 (2007); corrections: 8. 374 (2008).

23 J. A. Misewich, R. Martel, Ph. Avouris, J. C. Tsang, S. Heinze, and J. Tersoff, Science 300, 783 (2003).

24 J. Chen, V. Perebeinos, M. Freitag, J. Tsang, Q. Fu, J. Liu, and Ph. Avouris, Science 310, 1171 (2005).

25 O. V. Kibis and M. E. Portnoi, Tech. Phys. Lett. 31, 671 (2005).

26 O. V. Kibis, M. Rosenau da Costa, and M. E. Portnoi, Nano Lett. 7, 3414 (2007).

27 K. G. Batrakov, P. P. Kuzhir, and S. A. Maksimenko, Proc. SPIE 6328, 63280Z (2006).

28 P. Kuzhir, K. Batrakov, S. Maksimenko, Synthesis and Reactivity in Inorganic, Metal-Organic and Nano-Metal Chemistry 37, 341 (2007)

${ }^{29}$ K. G. Batrakov, P. P. Kuzhir, and S. A. Maksimenko, Physica E 40, 1065 (2008).

30 G. Miano and F. Villone, IEEE Trans. Antennas Propagat. 54, 2713 (2006).

31 J. Hao and G. W. Hanson, Phys. Rev. B 74, 035119 (2006).

32 J. Hao and G. W. Hanson, Phys. Rev. B 75, 165416 (2007).

33 M. V. Shuba, S. A. Maksimenko, and A. Lakhtakia, Phys. Rev. B 76, 155407 (2007).

${ }^{34}$ Y. Huang, W.-Y. Yin, and Q. H. Liu, IEEE Trans. Nanotechnol. 7, 331 (2008).

35 Y. Lan, B. Zeng, H. Zhang, B. Chen, and Z. Yang, Int. J. Infrared Millim. Waves 27, 871 (2006).

36 S. Bandow, M. Takizawa, K. Hirahara, M. Yudasaka, and S. Iijima, Chem. Phys. Lett. 337, 48 (2001).

37 S. Iijima, Nature (London) 354, 56 (1991).

38 M. Ge and K. Sattler, Science 260, 515 (1993).

39 S. Wang and M. Grifoni, Phys. Rev. Lett. 95, 266802 (2005). 
40 R. Saito, G. Dresselhaus, and M. S. Dresselhaus, Appl. Phys. 73, 494 (1993).

41 P. Lambin, V. Meunier, and A. Rubio, Phys. Rev. B 62, 5129 (2000).

42 K.-H. Ahn,Y.-H. Kim, J. Wiersig, and K. J. Chang, Phys. Rev. Lett. 90, 026601 (2003).

43 Y.-G. Yoon, P. Delaney, and S. G. Louie, Phys. Rev. B 66, 073407 (2002).

44 A. M. Lunde, K. Flensberg, and A.-P. Jauho, Phys. Rev. B 71, 125408 (2005).

45 Y. H. Ho, G. W. Ho, S. C. Chen, J. H. Ho, and M. F. Lin, Phys. Rev. B 76, 115422 (2007).

46 Y.-K. Kwon and D. Tomanek, Phys. Rev. B 58, R16001 (1998).

47 A. A. Abrikosov, D. V. Livanov, and A. A. Varlamov, Phys. Rev. B 71, 165423 (2005).

48 P. N. Dyachkov and D. V. Makaev, Phys. Rev. B 74, 155442 (2006).

49 M. A. Tunney and N. R. Cooper, Phys. Rev. B 74, 075406 (2006).

50 B. Bourlon, C. Miko, L. Forro, D. C. Glattli, and A. Bachtold, Phys. Rev. Lett. 93, 176806 (2004).

51 C. A. Balanis, Antenna Theory: Analysis and Design (Wiley, New York, NY, USA, 1997).

52 S. A. Maksimenko, A. A. Khrushchinsky, G. Ya. Slepyan, and O. V. Kibis, J. Nanophoton. 1, 013505 (2007).

53 A. S. Ilyinsky, G. Ya. Slepyan, and A. Ya. Slepyan, Propagation, Scattering and Dissipation of Electromagnetic Waves (Peter Peregrinus, London, United Kingdom, 1993).

${ }^{54}$ L. Novotny and B. Hecht, Principles of Nano-optics (Cambridge University Press, Cambridge, United Kingdom,
2006).

55 D. Colton and R. Kress, Integral Equation Methods in Scattering Theory (Wiley, New York, NY, USA, 1983).

56 E. Wolf, Astrophys. Space Sci. 227, 277 (1995).

57 M. Born and E. Wolf, Principles of Optics (Pergamon, Oxford, 1999).

58 R. W. P. King and T. T. Wu, IEEE Trans. Antennas Propagat. 14, 524 (1966).

59 C.-K. Lee, J. Cho, J. Ihm, and K.-H. Ahn, Phys. Rev. B 69, 205403 (2004).

60 F. L. Shyu and M. F. Lin, Phys. Rev. B 62, 8508 (2000).

61 J. M. Pitarke, V. M. Silkin, E. V. Chulkov, and P. M. Echenique, Rep. Prog. Phys. 70, 1 (2007).

62 L. Novotny, Phys. Rev. Lett. 98, 266802 (2007).

63 A. L. Elias, J. A. Rodriguez-Manzo, M. R. McCartney, D. Golberg, A. Zamudio, S. E. Baltazar, F. Lopez-Urias, E. Munoz-Sandoval, L. Gu, C. C. Tang, D. J. Smith, Y. Bando, H. Terrones, and M. Terrones, Nano Lett. 5, 467 (2005).

64 G. W. Hanson, IEEE Trans. Antennas Propagat. 54, 76 (2006).

65 P. B. Johnson and R. W. Christy, Phys. Rev. B 12, 4370 (1972).

66 B. Wang, S. Yin, G. Wang, A. Buldum, and J. Zhao, Phys. Rev. Lett. 86, 2046 (2001).

67 K. C. Chin, A. Gohel, W. Z. Chen, H. I. Elim, W. Ji, G. L. Chong, C. H. Sow, and A. T. S. Wee, Chem. Phys. Lett. 409, 85 (2005).

68 Selected Papers on Linear Optical Composite Materials (A. Lakhtakia, ed.), (SPIE Press, Bellingham, WA, 1996). 